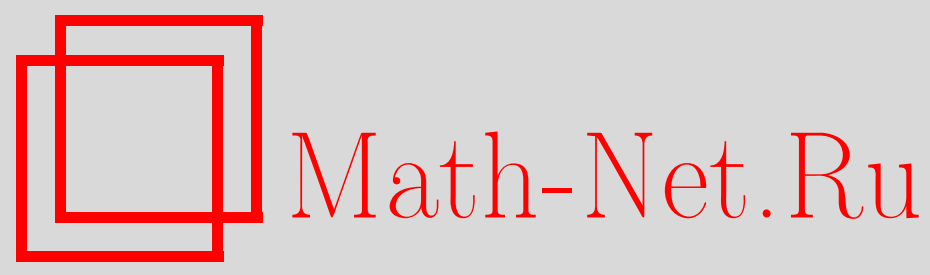

Ю. М. Нечепуренко, Оценка нормы матрицы Грина через интегральный критерий качества дихотомии и границы хаусдорфова множества, Матем. заметки, 2002, том 71, выпуск $2,232-238$

DOI: https://doi.org/10.4213/mzm342

Использование Общероссийского математического портала Math-Net.Ru подразумевает, что вы прочитали и согласны с пользовательским соглашением http://www . mathnet.ru/rus/agreement

Параметры загрузки:

IP: 52.6 .47 .48

26 апреля 2023 г., 16:07:02

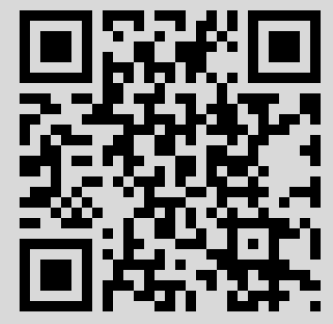


тОм 71 выПУСК 2 фЕВРАль 2002

УДК 512.64

\section{ОЦЕНКА НОРМЫ МАТРИЦЫ ГРИНА \\ ЧЕРЕЗ ИНТЕГРАЛЬНЫЙ КРИТЕРИЙ КАЧЕСТВА ДИХОТОМИИ \\ И ГРАНИЦЫ ХАУСДОРФОВА МНОЖЕСТВА \\ Ю. М. Нечепуренко}

Для системы обыкновенных дифференциальных уравнений вида $d u / d t=A u+f(t)$, $-\infty<t<\infty$, получены новые верхние оценки второй нормы матрицы Грина через интегральый критерий качества дихотомии и границы хаусдорфова множества матрицы $A$. Эти оценки значительно лучше известных для многих приложений.

Библиография: 6 названий.

Пусть $A$ - квадратная матрица порядка $n$ такая, что мнимая ось не содержит точек ее спектра и разбивает его на два непустых подмножества, лежащих соответственно в левой и правой полуплоскостях. Величину $\theta=\|H\|_{2}$, где

$$
H=\frac{1}{2 \pi} \int_{-\infty}^{\infty}(i \omega I-A)^{*}(i \omega I-A)^{-1} d \omega
$$

а $I$ означает единичную матрицу порядка $n$, называют интегральным критерием качества дихотомии спектра матрицы $A$ мнимой осью [1].

Обозначим через $P$ спектральньй проектор (проектор Рисса), отвечающий части спектра матрицы $A$, лежащей в левой полуплоскости. В работе [2] было показано, что

$$
\begin{aligned}
&\|\exp \{t A\}(I-P)\|_{2} \leqslant \sqrt{c_{1}} \exp \left\{\frac{t}{2 \theta}\right\}, t \leqslant 0 \\
&\|\exp \{t A\} P\|_{2} \leqslant \sqrt{c_{2}} \exp \left\{-\frac{t}{2 \theta}\right\}, \quad t \geqslant 0
\end{aligned}
$$

где

$$
c_{1}=2 h_{\max } \theta, \quad c_{2}=2\left|h_{\min }\right| \theta,
$$

a $h_{\min }=h_{\min }(A)<0$ и $h_{\max }=h_{\max }(A)>0$ означают соответственно левую и правую гранищы хаусдорфова множества

$$
W(A)=\left\{\frac{(A v, v)}{(v, v)}: v \in \mathbb{C}^{n}, v \neq 0\right\}
$$

Работа выполнена при финансовой поддержке Российского фонда фундаментальных исследований, грант № 01-01-00269. 
матрицы $A$ :

$$
h_{\min }(A)=\min \{\operatorname{Re} \lambda: \lambda \in W(A)\}, \quad h_{\max }(A)=\max \{\operatorname{Re} \lambda: \lambda \in W(A)\} .
$$

Неравенства (2), (3) являются верхними оценками нормы матрицы Грина [3], [4]

$$
G(t)=\frac{1}{2 \pi} \int_{-\infty}^{\infty}(i \omega I-A)^{-1} \exp \{i \omega t\} d \omega=\exp \{t A\} \times \begin{cases}P-I, & t<0 \\ P, & t>0\end{cases}
$$

системы обыкновенньх дифференциальных уравнений

$$
\frac{d u}{d t}=A u+f(t), \quad-\infty<t<\infty
$$

и имеют много других полезных приложений. В частности, они позволяют оценить норму спектрального проектора:

$$
\|P\|_{2}=\|I-P\|_{2} \leqslant \sqrt{\min \left\{c_{1}, c_{2}\right\}}
$$

и для решения $u(t)=\exp \{t A\} u^{0}$ задачи Коши

$$
u(0)=u^{0}, \quad \frac{d u}{d t}=A u
$$

оценить скорость убьвания жесткой компоненты по сравнению с главной:

$$
\frac{\|P u(t)\|_{2}}{\|(I-P) u(t)\|_{2}} \leqslant \sqrt{c_{1} \mathrm{c}_{2}} \exp \left\{-\frac{t}{\theta}\right\} \frac{\left\|P u^{0}\right\|_{2}}{\left\|(I-P) u^{0}\right\|_{2}}, \quad t \geqslant 0 .
$$

Действительно, из неравенств (2), (3) следует, что для любого $u^{0} \in \mathbb{C}^{n}$ и $t \geqslant 0$ выполнены оценки

$$
\begin{gathered}
\left\|\exp \{-t A\}(I-P) u^{0}\right\|_{2} \leqslant \sqrt{c_{1}} \exp \left\{-\frac{t}{2 \theta}\right\}\left\|(I-P) u^{0}\right\|_{2}, \\
\left\|\exp \{t A\} P u^{0}\right\|_{2} \leqslant \sqrt{c_{2}} \exp \left\{-\frac{t}{2 \theta}\right\}\left\|P u^{0}\right\|_{2} .
\end{gathered}
$$

Подставив в (7a) $\exp \{t A\} u^{0}$ вместо $u^{0}$ и разделив левую (правую) части неравенства $(7 б)$ на соответственно правую (левую) части полученного неравенства, приходим к (6).

Основным результатом данной работы является

Теорема 1. Оиенки (2), (3) справедливы с константами

$$
c_{1}=\frac{1}{\gamma}\left(\frac{\alpha+\gamma}{\alpha+\beta}\right)^{1+\alpha}, \quad c_{2}=\frac{1}{\gamma}\left(\frac{\beta+\gamma}{\alpha+\beta}\right)^{1+\beta},
$$

где $\alpha=1 /\left(2\left|h_{\min }\right| \theta\right), \beta=1 /\left(2 h_{\max } \theta\right), \gamma=\max \{\alpha, \beta\}$.

Отсюда, учитьвая, что $\gamma \leqslant 1$ в силу неравенства (5) с константами (4), несложно вывести следующую оценку нормы матрицы Грина. 
СЛЕДСТВИЕ. Имеет место неравенство

$$
\|G(t)\|_{2} \leqslant \sqrt{c} \exp \left\{-\frac{|t|}{2 \theta}\right\}, \quad-\infty<t<\infty,
$$

2de

$$
c=\frac{1}{\gamma}\left(\frac{2 \gamma}{\alpha+\beta}\right)^{1+\gamma} \leqslant \frac{4}{\gamma}=8 \min \left\{h_{\max },\left|h_{\min }\right|\right\} \theta .
$$

Отметим, что константы $c_{1}$ и $c_{2}$ из теоремы 1 не превосходят одноименных констант (4) и значительно меньше их в случаях, когда либо $h_{\max } \gg\left|h_{\min }\right|$, либо $\left|h_{\min }\right| \gg h_{\max }$. Действительно, при $\delta>0$ функция $\varphi(x)=(\delta+x)^{1+\delta} / x$ убывает в интервале $0<x \leqslant 1$ и, следовательно,

$$
\frac{1}{\gamma}\left(\frac{\alpha+\gamma}{\alpha+\beta}\right)^{1+\alpha} \leqslant \frac{1}{\beta}=2 h_{\max } \theta, \quad \frac{1}{\gamma}\left(\frac{\beta+\gamma}{\alpha+\beta}\right)^{1+\beta} \leqslant \frac{1}{\alpha}=2\left|h_{\min }\right| \theta .
$$

С другой стороны, если $h_{\max } \geqslant\left|h_{\min }\right|$, то $\gamma=\alpha$ и

$$
\frac{1}{\gamma}\left(\frac{\alpha+\gamma}{\alpha+\beta}\right)^{1+\alpha}=\frac{1}{\alpha}\left(\frac{2 \alpha}{\alpha+\beta}\right)^{1+\alpha} \leqslant \frac{4}{\alpha}=8\left|h_{\min }\right| \theta
$$

а если $\left|h_{\min }\right| \geqslant h_{\max }$, то $\gamma=\beta$ и

$$
\frac{1}{\gamma}\left(\frac{\beta+\gamma}{\alpha+\beta}\right)^{1+\beta}=\frac{1}{\beta}\left(\frac{2 \beta}{\alpha+\beta}\right)^{1+\beta} \leqslant \frac{4}{\beta}=8 h_{\max } \theta .
$$

Таким образом, константы из теоремы 1 удовлетворяют следующим неравенствам:

$$
c_{1} \leqslant \min \left\{2 h_{\max }, 8\left|h_{\min }\right|\right\} \theta, \quad c_{2} \leqslant \min \left\{8 h_{\max }, 2\left|h_{\min }\right|\right\} \theta .
$$

Если матрица $A$ является конечномерным аналогом линейного неограниченного замкнутого оператора $L$, действующего в сепарабельном гильбертовом пространстве $\mathscr{H}$, с областью определения $D(L) \subset \mathscr{H}$, то для того чтобы оценки (2), (3) с константами (4) оставались нетривиальными при $n \rightarrow \infty$, необходимо и достаточно, чтобы хаусдорфово множество оператора $L$ было ограничено слева и справа, т.е.

$$
\begin{aligned}
& h_{\text {min }}(L)=\inf \left\{\operatorname{Re} \frac{(L x, x)_{\mathscr{H}}}{(x, x)_{\mathscr{H}}}: x \in D(L), x \neq 0\right\}>-\infty, \\
& h_{\text {max }}(L)=\sup \left\{\operatorname{Re} \frac{(L x, x)_{\mathscr{H}}}{(x, x) \mathscr{H}}: x \in D(L), x \neq 0\right\}<\infty,
\end{aligned}
$$

и был ограничен интегральный критерий качества дихотомии спектра оператора $L$ мнимой осью:

$$
\sup _{x \in \mathscr{H}} \frac{1}{2 \pi} \int_{-\infty}^{\infty} \frac{\left\|(i \omega-L)^{-1} x\right\|_{\mathscr{H}}^{2}}{\|x\|_{\mathscr{H}}^{2}} d \omega<\infty .
$$

В то же время, как это следует из неравенств (9), для нетривиальности при $n \rightarrow \infty$ оценок из теоремы 1 необходимо и достаточно вьполнения условия (12) и какого-нибудь 
одного из условий (10), (11). Учитывая, что для многих задач математической физики, таких как уравнения конвекции-диффузии, переноса нейтронов и т.п., условие (11) выполнено, а условие (10) - нет, очевидно не только количественное, но и качественное преимущество оценок из теоремы 1 по сравнению с оценками (2), (3), (4).

Для доказательства теоремы 1 существенно предположение о том, что мнимая ось разбивает спектр матрицы $A$ на два непустых подмножества. Поэтому полученные оценки нельзя использовать в случаях, когда $P=0$ или $I$. Для этих случаев, требующих отдельного рассмотрения, оценки, аналогичные оценкам из теоремы 1 , получены в работе [5].

ДоКАЗАТЕЛЬСТво тЕОРЕМЫ 1 . Учитьвая, что $P(-A)=I-P(A), H(-A)=H(A)$,

$$
h_{\min }(-A)=-h_{\max }(A), \quad h_{\max }(-A)=-h_{\min }(A),
$$

нетрудно проверить, что неравенство (2), (8) эквивалентно неравенству (3), (8), примененному к матрице $-A$. Таким образом, для доказательства теоремы достаточно доказать справедливость одного из этих неравенств. Докажем, например, неравенство (3), (8).

Известно [1], [6], что матричный интеграл (1) является решением обобщенного уравнения Ляпунова, которое в наших обозначениях имеет следующий вид:

$$
A^{*} H+H A=(I-P)^{*}(I-P)-P^{*} P, \quad H P=P^{*} H .
$$

Если матрища $A$ не имеет собственных значений на мнимой оси, то система матричных уравнений (14) имеет единственное эрмитовое положительно определенное решение $H=H^{*}>0$. Для этого решения справедливо представление (1) и представление

$$
H=\int_{0}^{\infty}\left[P^{*} \exp \left\{s A^{*}\right\} \exp \{s A\} P+(I-P)^{*} \exp \left\{-s A^{*}\right\} \exp \{-s A\}(I-P)\right] d s .
$$

Пусть $u^{0}$ - произвольньй вектор длины $n, u=\exp \{t A\} u^{0}, \widetilde{H}=P^{*} H P$. Домножив первое уравнение (14) слева на $P^{*}$ и справа на $P$, получим равенство

$$
A^{*} \widetilde{H}+\widetilde{H} A=-P^{*} P \text {, }
$$

в силу которого

$$
\frac{d}{d t}(\widetilde{H} u, u)=\left(\left(A^{*} \widetilde{H}+\widetilde{H} A\right) u, u\right)=-(P u, P u) \leqslant-\frac{1}{\theta}(H P u, P u)=-\frac{1}{\theta}(\widetilde{H} u, u) .
$$

Следовательно,

$$
(\widetilde{H} u, u) \leqslant \exp \left\{-\frac{t}{\theta}\right\}\left(\widetilde{H} u^{0}, u^{0}\right), \quad t \geqslant 0 .
$$

Отсюда для произвольного $\tau$ такого, что $t \geqslant \tau \geqslant 0$, имеем

$$
\left(\exp \left\{-\tau A^{*}\right\} \widetilde{H} \exp \{-\tau A\} u, u\right) \leqslant \exp \left\{-\frac{t-\tau}{\theta}\right\}\left(\widetilde{H} u^{0}, u^{0}\right) .
$$


В силу представления (15)

$$
\begin{aligned}
\left(\exp \left\{-\tau A^{*}\right\} \tilde{H} \exp \{-\tau A\} u, u\right) & =\int_{-\tau}^{\infty}\left(\exp \left\{s A^{*}\right\} \exp \{s A\} P u, P u\right) d s \\
& \geqslant \int_{-\tau}^{\infty} \sigma(s)^{2} d s\|P u\|_{2}^{2}
\end{aligned}
$$

где $\sigma(s)$ - минимальное сингулярное число матрицы $\exp \{s A\}$. С другой стороны, в силу того же представления $\widetilde{H} \leqslant H$ и, следовательно,

$$
\left(\widetilde{H} u^{0}, u^{0}\right) \leqslant \theta\left\|u^{0}\right\|_{2}^{2}
$$

Используя неравенства (17) и (18) для оценки соответственно левой и правой частей неравенства (16), приходим к следующему неравенству:

$$
\left\|\exp \{t A\} P u^{0}\right\|_{2}^{2} \leqslant \theta \exp \left\{\frac{\tau}{\theta}\right\}\left(\int_{-\tau}^{\infty} \sigma(s)^{2} d s\right)^{-1} \exp \left\{-\frac{t}{\theta}\right\}\left\|u^{0}\right\|_{2}^{2},
$$

справедливому при всех $t \geqslant \tau \geqslant 0$.

В силу известного [1] неравенства

$$
\|\exp \{t A\}\|_{2} \leqslant \exp \left\{h_{\max } t\right\}, \quad t \geqslant 0
$$

и второго равенства (13) для минимального сингулярного числа матричной экспоненты справедливы следующие нижние оценки:

$$
\sigma(s)=\frac{1}{\|\exp \{-s A\}\|_{2}} \geqslant \begin{cases}\exp \left\{h_{\min } s\right\}, & s \geqslant 0 \\ \exp \left\{h_{\max } s\right\}, & s \leqslant 0\end{cases}
$$

Отсюда

$$
\begin{aligned}
\int_{-\tau}^{\infty} \sigma(s)^{2} d s & \geqslant \int_{0}^{\infty} \exp \left\{2 h_{\min } s\right\} d s+\int_{-\tau}^{0} \exp \left\{2 h_{\max } s\right\} d s \\
& =\frac{1}{2\left|h_{\min }\right|}+\frac{1-\exp \left\{-2 h_{\max } \tau\right\}}{2 h_{\max }}=\theta\left(\alpha+\beta-\beta \exp \left\{-\frac{\tau}{\beta \theta}\right\}\right) .
\end{aligned}
$$

Подставив эту оценку в правую часть неравенства (19), получим, что при всех $t \geqslant \tau \geqslant 0$ и $u^{0} \in \mathbb{C}^{n}$ справедливо неравенство

$$
\left\|\exp \{t A\} P u^{0}\right\|_{2}^{2} \leqslant c(\tau) \exp \left\{-\frac{t}{\theta}\right\}\left\|u^{0}\right\|_{2}^{2}
$$

и, следовательно, справедливо неравенство

$$
\|\exp \{t A\} P\|_{2}^{2} \leqslant c(\tau) \exp \left\{-\frac{t}{\theta}\right\}, \quad t \geqslant \tau \geqslant 0
$$

где

$$
c(\tau)=\exp \left\{\frac{\tau}{\theta}\right\}\left(\alpha+\beta-\beta \exp \left\{-\frac{\tau}{\beta \theta}\right\}\right)^{-1}
$$


Отметим, что положив в $(22) \tau=0$, получим неравенство $(3),(4)$, а применив полученное неравенство к матрице $-A$, получим неравенство $(2),(4)$.

Наряду с оценкой $(22)$ в силу неравенства $\|P\|_{2} \leqslant 1 / \gamma$ и оценки (20) справедлива оценка

$$
\|\exp \{t A\} P\|_{2}^{2} \leqslant \frac{1}{\gamma} \exp \left\{\frac{t}{\beta \theta}\right\}, \quad t \geqslant 0 .
$$

Правая часть неравенства (22) убывает с ростом $t$, а правая часть неравенства (23) возрастает. Следовательно, если в качестве $\tau$ выбрать решение уравнения

$$
c(\tau) \exp \left\{-\frac{\tau}{\theta}\right\}=\frac{1}{\gamma} \exp \left\{\frac{\tau}{\beta \theta}\right\}, \quad \tau \geqslant 0,
$$

то оценка (22) будет справедлива при всех $t \geqslant 0$. Непосредственно проверяется, что

$$
\tau=\tau_{*}=\beta \theta \ln \frac{\beta+\gamma}{\alpha+\beta}
$$

является решением уравнения (24) и

$$
c\left(\tau_{*}\right)=\frac{1}{\gamma}\left(\frac{\beta+\gamma}{\alpha+\beta}\right)^{1+\beta} .
$$

Теорема 1 доказана.

Из теоремы 1 следуют неравенства (7) с константами $c_{1}$ и $c_{2}$, определенными в этой теореме. Однако по аналогии с доказательством теоремы 1 можно непосредственно вывести неравенства (7) с несколько меньшими константами.

ТЕОРема 2. Оиенки (7) справедливы с константами

$$
c_{1}=\left(\frac{\alpha+1}{\alpha+\beta}\right)^{1+\alpha}, \quad c_{2}=\left(\frac{\beta+1}{\alpha+\beta}\right)^{1+\beta}
$$

где $\alpha$ и $\beta$ определены как в теореме 1.

ДокАЗАТЕльство. В силу неравенств $(20)$ и $(21)$ для любого $u^{0} \in \mathbb{C}^{n}$ имеем

$$
\left\|\exp \{t A\} P u^{0}\right\|_{2}^{2} \leqslant \exp \left\{\frac{t}{\beta \theta}\right\}\left\|P u^{0}\right\|_{2}^{2}
$$

при всех $t \geqslant 0$ и

$$
\left\|\exp \{t A\} P u^{0}\right\|_{2}^{2} \leqslant c(\tau) \exp \left\{-\frac{t}{\theta}\right\}\left\|P u^{0}\right\|_{2}^{2}
$$

при всех $t \geqslant \tau \geqslant 0$. Правая часть неравенства (26) возрастает, а правая часть неравенства (27) убывает с ростом $t$. Поэтому если в качестве $\tau$ выбрать решение

$$
\tau_{0}=\beta \theta \ln \frac{\beta+1}{\alpha+\beta}
$$


уравнения

$$
c(\tau) \exp \left\{-\frac{\tau}{\theta}\right\}=\exp \left\{\frac{\tau}{\beta \theta}\right\}, \quad \tau \geqslant 0,
$$

то оценка (27) будет справедлива при всех $t \geqslant 0$. Учитывая, что

$$
c\left(\tau_{0}\right)=\left(\frac{\beta+1}{\alpha+\beta}\right)^{1+\beta}
$$

приходим к оценке (7б), (25). Справедливость оценки (7а), (25) следует из оценки (7б), (25), примененной к матрице $-A$.

Отметим, что функция $c(\tau)$ убьвает в интервале $0 \leqslant \tau \leqslant \tau_{0}$ и возрастает при $\tau \geqslant \tau_{0}$. Следовательно, выбор $\tau<\tau_{*}$ и $\tau<\tau_{0}$ при получении из оценки (21) оценок вида $(3)$ и (7б) привел бы к более грубому результату, чем $\tau=\tau_{*}$ и $\tau_{0}$ соответственно.

\section{СПИСОК ЦИТИРОВАННОЙ ЛИТЕРАТУРЫ}

[1] Годунов С. К. Современные аспекты линейной алгебры. Новосибирск: Научная книга, 1997.

[2] Годунов С. К., Нечепуренко Ю. М. Оценки для главной и жесткой компонент на основе интегрального критерия качества дихотомии // ЖВМиМФ. 2000. Т. 40. №1. С. 35-42.

[3] Далецкий Ю.А., Крейн М.Г. Устойчивость решений дифференциальных уравнений в банаховом пространстве. М.: Наука, 1970.

[4] Годунов С. К. Обыкновенные дифференциальные уравнения с постоянными коэффициентами. Новосибирск: Изд-во НГУ, 1994.

[5] Нечепуренко Ю. М. Об оценке нормы матричной экспоненты // Докл. РАН. 2001. Т. 377. №5. C. 597-600.

[6] Булгаков А.Я. Обоснование гарантированной точности выделения инвариантных подпространств несамосопряженных матриц // Тр. ИМ СО АН СССР. Т. 15. Новосибирск, 1989. C. $12-92$. 